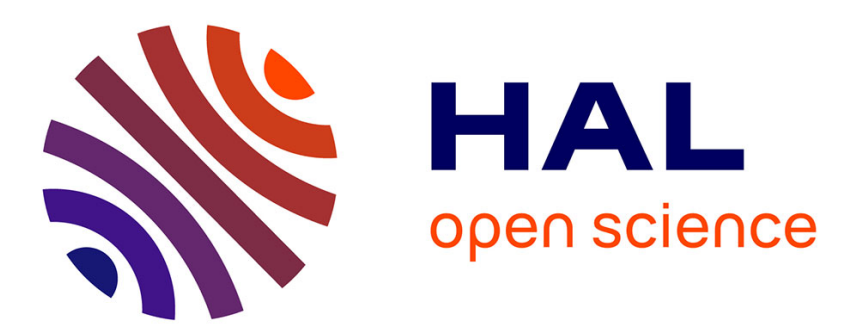

\title{
A three components model for simplified building scattering in urban environment
}

M Ait-Ighil, F Pérez-Fontán, J Lemorton, F Lacoste, C Bourga, M Bousquet

\section{To cite this version:}

M Ait-Ighil, F Pérez-Fontán, J Lemorton, F Lacoste, C Bourga, et al.. A three components model for simplified building scattering in urban environment. 6th European Conference on Antennas and Propagation (EUCAP 2012), May 2012, PRAGUE, Czech Republic. pp.2449 - 2453, 10.1109/EuCAP.2012.6206588 . hal-01080654

\section{HAL Id: hal-01080654 https://hal.science/hal-01080654}

Submitted on 5 Nov 2014

HAL is a multi-disciplinary open access archive for the deposit and dissemination of scientific research documents, whether they are published or not. The documents may come from teaching and research institutions in France or abroad, or from public or private research centers.
L'archive ouverte pluridisciplinaire HAL, est destinée au dépôt et à la diffusion de documents scientifiques de niveau recherche, publiés ou non, émanant des établissements d'enseignement et de recherche français ou étrangers, des laboratoires publics ou privés. 


\title{
A Three Components Model for Simplified Building Scattering in Urban Environment
}

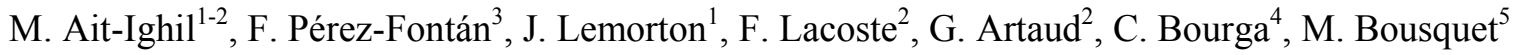 \\ ${ }^{1}$ ONERA - The French Aerospace Lab, DEMR/RCP, 2 Avenue Edouard Belin, 31055 Toulouse, France. \\ mehdi.ait_ighil@onera.fr,joel.lemorton@onera.fr \\ ${ }^{2}$ CNES Toulouse, 18 Avenue Edouard Belin, 31400 Toulouse, France. \\ frederic.lacoste@cnes.fr,geraldine.artaud@cnes.fr \\ ${ }^{3}$ Universidad de Vigo, 36310 Vigo, Spain. \\ fpfontan@tsc.uvigo.es \\ ${ }^{4}$ Thales Alenia Space, 26 Avenue J.F. Champollion, 31100 Toulouse, France. \\ christophe.bourga@thalesaleniaspace.com \\ ${ }^{5}$ ISAE Campus Supaéro, 10 Avenue Edouard Belin, 31055 Toulouse, France. \\ michel.bousquet@isae.fr
}

\begin{abstract}
Multipath represents a common predominant and uncontrolled component on channel impairments for land mobile satellite communication and navigation systems. Delayed signal replicas highly impact performances on communication systems while they induce strong positioning errors for navigation systems. The actual trend is to improve multipath wideband representation by using hybrid physical-statistical channel simulator instead of narrowband statistical approaches. In this context, this paper presents a new simplified model - Three Component Model - to represent building scattering in an efficient way which strongly improves time performances. This model is based on a fast formulation of Physical Optics to model specular reflections, backscattering and incoherent scattering from complex façades.
\end{abstract}

Keywords - Channel modelling, LMS systems, Physical Optics, Simplified Building Scattering, Multipaths, GNSS.

\section{INTRODUCTION}

Over the past decades, mobile applications using satellites have experienced a continuous growth and the demand for such systems does not fall off. Among all developed applications, satellites have first democratised voice and message transmission and more recently multimedia broadcasting with several international standards and satellite navigation systems as GPS (Global Positioning System) and future European Galileo system. The above systems are operating at L and Sband and are particularly sensitive to various channel impairments induced by urban environments, namely shadowing, multipath fading, delayed echoes, Doppler spreading, depolarization, etc. For wideband applications, a particular emphasis has to be placed on MP (Multipath) characterisation.

Many models have been proposed to represent the LMS (Land Mobile Satellite) propagation channel where both accuracy and computational efficiency are key parameters. All LMS channel models usually include two main aspects: the description of the propagation environment and the modelling of EM (ElectroMagnetic) interactions. All techniques can be grouped into three main approaches, namely, statistical, deterministic and hybrid physical-statistical, depending on how the environment and the interactions are modelled.

Statistical approaches [1] and state oriented models [2] present the advantage of being very fast with a low computational complexity. However, they are limited to narrowband modelling without neither MP delay repartition nor AoA (Angle of Arrival). Such methods are then not adapted for wideband communications and navigation issues. On the opposite side, deterministic approaches and asymptotic methods [3] are perfectly suitable for such problematic. The main advantage of asymptotic methods is their rich variety of outputs, e.g. polarisation, AoA, Doppler, delay, and the overall accuracy of their results. However, the implementation of such methods is complex, simulations are site-specific and time consuming while results accuracy is very dependent on environment representation, e.g. details, geometry of the scene or materials. From those statements, hybrid approaches [4] [5] [6] present the best compromise in terms of output diversity, accuracy, execution time and site flexibility. To achieve the site flexibility, hybrid approaches are usually based on a virtual city concept [7] made up from macro-statistics of a particular environment, e.g. urban, sub-urban or rural. Faster compared to deterministic approaches, they lie on simplified EM model to reproduce interactions between wave fronts and objects in the scene.

The work here presented is part of a wider project aiming at reproducing channel impairments in an enhanced LMS channel simulator. A specific attention has to be paid on the wideband representation of the channel and on the calculation time to achieve almost real time synthetic impulse response generation. As presented above, hybrid approaches are the most suitable for fulfilling such expectations. Figure 1 represents the retained architecture of the global channel simulator.

In this paper, only the simplified model for EM interactions on buildings is presented. The remainder of this paper is organized as follows: Section II presents the fundamentals of the simplified EM model from simulation observations to the 
main principle of the Three Component Model. Section III presents one validation example on a complex and isolated building using MoM (Method of Moments) as a reference. Finally, Section IV concludes and presents some leads on features to be added to improve the actual implemented model.

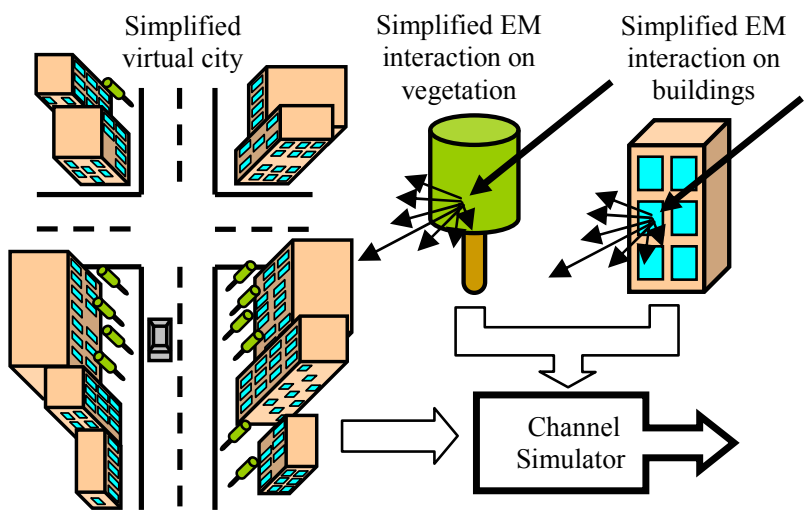

Figure 1. Architecture of the global channel simulator

\section{THE THREE COMPONENT MODEL}

\section{A. Simulation observations}

The Three Component Model (TCM) has been first imagined observing simulation results using independent numerical tools during different works. The first observation, Figure 2, has been made using Elsem3D during work presented in [8]. Elsem3D is a research code developed by ONERA, based on EFIE (Electric Field Integral Equation) and solved with MoM techniques in the frequency domain. Figure 2 represents the normalised narrowband power scattered by a complex façade when a receiver is passing by at a distance of $\mathrm{X}=10 \mathrm{~m}$. The receiver trajectory is collinear to the building with $\mathrm{Y}= \pm 100 \mathrm{~m}$. The satellite is at $2010^{6} \mathrm{~m}$, elevation $40^{\circ}$, and azimuth $40^{\circ}$. The complex façade used is placed at $\mathrm{Y}=0 \mathrm{~m}$. Its geometry is presented in Figure 3 and detailed in [8].

As visible on Figure 2, the scattering pattern can be divided in 2 zones: $Y= \pm 20 \mathrm{~m}$ where the building has an influence on the scattered power, and out of this interval where the façade has no influence. In the no influence zone, the power scattered by the façade rapidly decreases with smooth variations. In the influence zone the scattered power can be divided into three regions. The first one represents a strong peak around $Y=-10 \mathrm{~m}$. The position of this contribution can be easily linked to the incidence of the satellite ( $40^{\circ}$ azimuth). This peak can be attributed to a specular phenomenon. A second peak is observable around $\mathrm{Y}=+10 \mathrm{~m}$. Slightly lower, this second contribution can be attributed to backscattering phenomena, symmetrical w.r.t the specular component but in the opposite azimuth direction. The influence zone of the façade is characterised by a power threshold around $-30 \mathrm{~dB}$ comparable to a background random floor due to bistatic scattering.

A second independent simulation result has confirmed what has been observed in Figure 2. Figure 4 has been extracted from results presented in [9] using the SE-RAY-EM/FERMAT software. This commercial tool is co-developed by ONERA and Oktal-SE Company. It is based on asymptotic methods
(GO (Geometrical Optics), PO (Physical Optics) and ECM (Equivalent Current Method)) using a SBR (Shooting and Bouncing Rays) algorithm. This software is able to compute complex channel impulse response considering power, phase, delay and AoA for each MP. Figure 4 represents the azimuthal power profile using $5^{\circ}$ beams at the receiver location. The context of simulation is the following: a mobile receiver is placed in a one sided urban canyon at a distance of $12 \mathrm{~m}$ from the buildings. The satellite is placed at elevation $50^{\circ}$ and azimuth $20^{\circ}$.

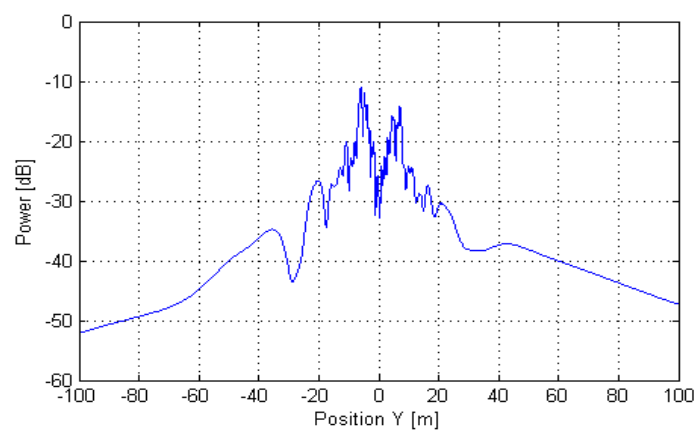

Figure 2. Normalised scattered powed received when passing close to a city center building type.

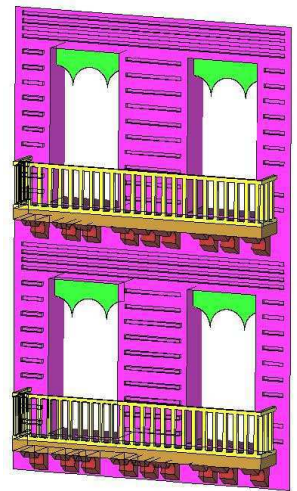

Figure 3. Geometry of complex city center building type

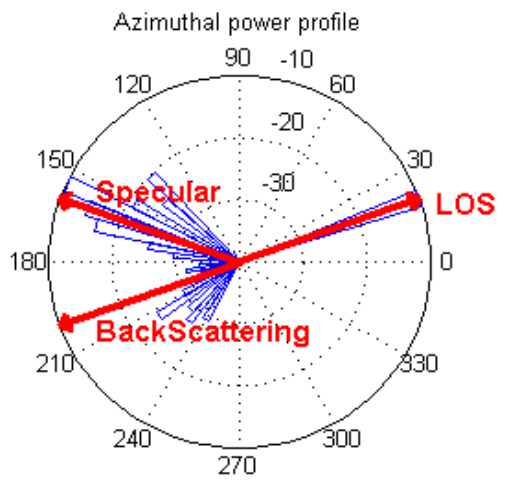

Figure 4. Azimuthal power profile at one receiver position.

As visible in Figure 4, the LOS signal comes from $\varphi=20^{\circ}$ with $0 \mathrm{~dB}$ power (normalised). Also noticeable, two zones of strong power contribution $\varphi=\left[110^{\circ} ; 170^{\circ}\right]$ and $\varphi=\left[210^{\circ} ; 240^{\circ}\right]$. Note that the second zone has lower power and spreading w.r.t the first zone. Using simple geometry relations, the first zone can be linked to a specular phenomenon $\left(\varphi=160^{\circ}\right.$, red arrow $)$ 
and the second to a backscattering phenomenon $\left(\varphi=200^{\circ}\right)$ coming from the opposite direction w.r.t. the LOS. The random phenomenon observed in Figure 2 is here hardly noticeable except for $\varphi=\left[170^{\circ} ; 200\right]$.

\section{B. Proposed approach}

Observations made in the previous section has highlighted that the scattering pattern of a complex building can be broken down into three different propagation phenomena namely, specular reflection, backscattering and incoherent scattering as presented in Figure 5 and described next. This model is therefore called the Three Component Model.

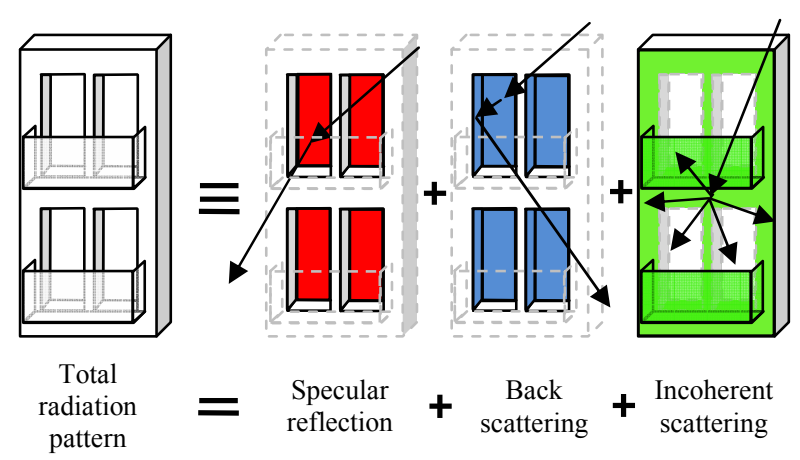

Figure 5. Decomposition principle of the Three Component Model

\section{1) Specular Reflection model}

The specular reflection phenomenon is originating from wide and smooth surfaces. Typical specular elements present on façades are windows and walls. Since the used frequency is in $\mathrm{L}$ or $\mathrm{S}$ band $(\lambda>20 \mathrm{~cm})$, the walls can be assumed to be smooth w.r.t the wave length. To refine the model, additional incoherent scattering can be added on wall surfaces. The internal EM implementation of the specular reflection model is based on a fast formulation of PO extracted from [10]. The fast denomination comes from the fact that the current integral does not have to be evaluated on the surface and is replaced by its analytical solution, eq(1).

$$
\sigma_{p q}^{0}=\frac{k_{0}^{2}}{\pi} L_{x} L_{y} \operatorname{sinc}\left(\frac{k_{0} \xi_{x} L_{x}}{2 \pi}\right) \operatorname{sinc}\left(\frac{k_{0} \xi_{y} L_{y}}{2 \pi}\right)\left|\alpha_{p q}\right|^{2}
$$

where $\sigma_{p q}^{0}$ denotes the normalised RCS for transmitting polarisation $p$ and receiving polarisation $q, k_{0}$ represents the wave number, $L_{x}$ and $L_{y}$ the size of the facet, $\xi_{x}$ and $\xi_{y}$ is an angular function depending of the direction of arrival of the wave fronts and the direction of departure to reach the receiver and $\alpha_{p q}$ a polarisation dependent loss term.

To obtain this analytical solution, the current integration has been performed on rectangular surfaces. To use this analytical formulation the final facets will have to be rectangular. Also due to $\mathrm{PO}$ formulation, the receiver position has to fulfil the far field condition w.r.t the facet size. This condition usually conducts to sub cut the façade into smaller facets. The major advantage of such formulation is the calculation time compared to classical PO. Analytically, this model is also suitable for various frequencies i.e. L, S and C bands, various dielectric materials and for polarimetric concerns.

\section{2) Backscattering model}

The backscattering phenomenon is originating from dihedral effects essentially taking place on window corners. Note that this phenomenon has also been observed by Lehner and al in [11]. The backscattering effect is responsible of significant contributions coming to the receiver from the exact opposite direction compared to LOS, as illustrated in Figure 4. The EM internal implementation of this model is for the moment based on the specular reflection model coupled with a pre-calculated form of GO. In a first approximation to reduce time consumption, only one of the two plates composing the dihedral is considered. This plate is normal to the global Y axis as presented in Figure 6 . The original incoming wave front (Tx) is replaced by its image (Tx') assuming a reflection on the $\mathrm{X}$ normal plate. The radiation of the Y-plate is then calculated using the specular reflection model adjusted by a coefficient 2 to compensate the lack of the second half of the dihedral which does not radiate. This implementation of the backscattering model seems to show limitations as presented and discussed in section III and will have to be improved.

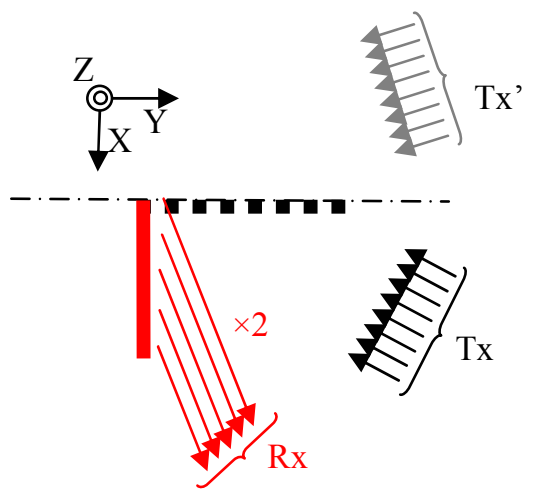

Figure 6. Principle of the backscattering model

\section{3) Incoherent Scattering model}

The incoherent scattering phenomenon is similar to a background random noise generated by the façade around itself. This phenomenon has been highlighted in Figure 2. It is due to all small architectural elements present on complex façades as guardrails or window decorations. The size of those objects is about one wave length or smaller so they scatter power almost uniformly in all directions. This phenomenon was also called "the influence zone of the façade" in section II.A. The EM implementation of the incoherent scattering model takes into account five different types of rough surfaces. The first four models are based on EM formulations extracted from [10]. They consider two different roughness profiles, slightly rough or very rough, each one being associated to two different roughness distributions, gaussian or exponential. Those four models are parameterised using two variables, the correlation length of the surface and the standard deviation of the roughness height. One last model has been implemented and is based on the specular reflection model where each elementary surface has a random reflection phase to break down the coherent reflection and consequently the specular phenomenon. 
Surface currents on city centre building type (Based on Elesem3D MoM)

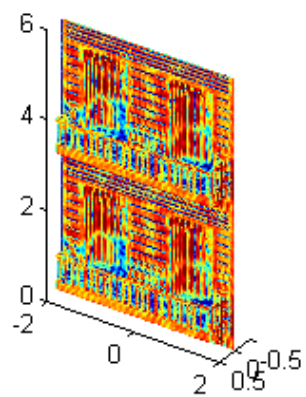

Simulated simplified city centre building type (Simplified model)

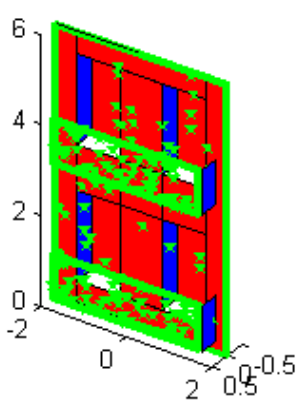

City centre type building

Comparison between exact and simplified methods

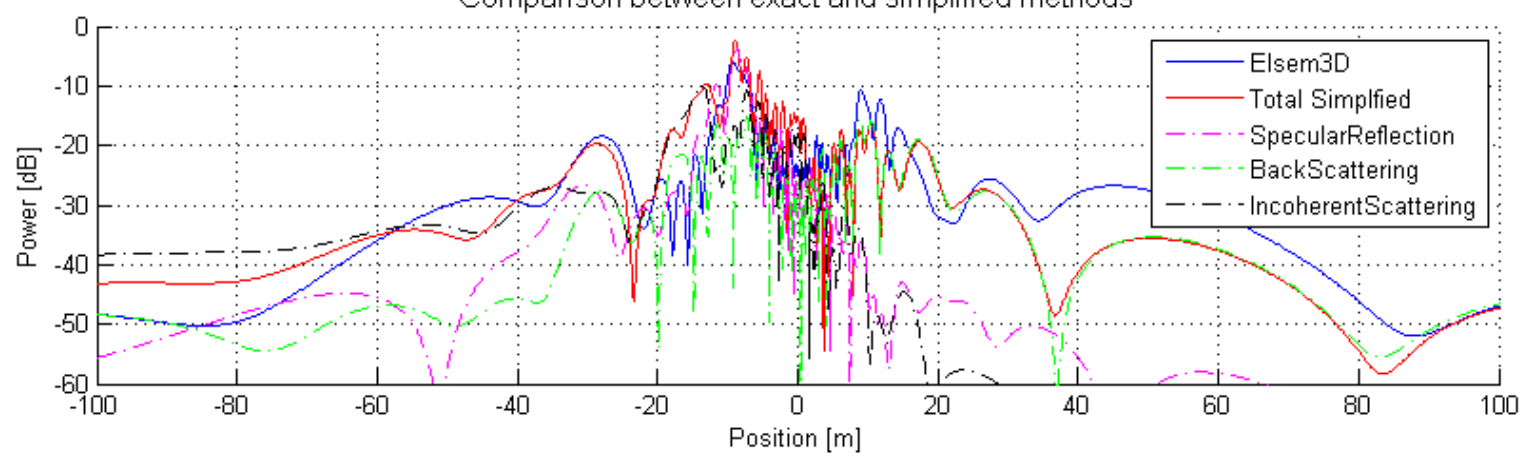

Figure 7. Decomposition of the total scattering of city centre building type, elevation $=0^{\circ}$, azimuth $=40^{\circ}$

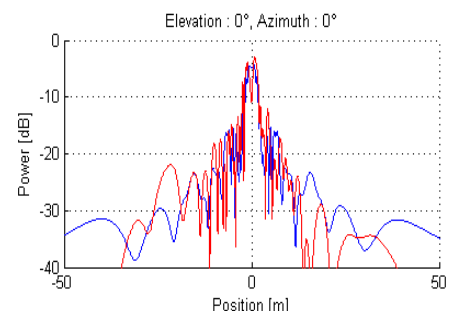

Elevation : $40^{\circ}$, Azimuth : $0^{\circ}$

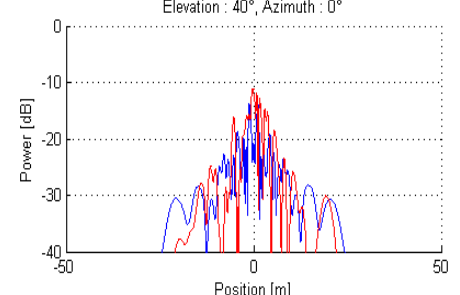

Evation : $80^{\circ}$, Azimuth : $0^{\circ}$

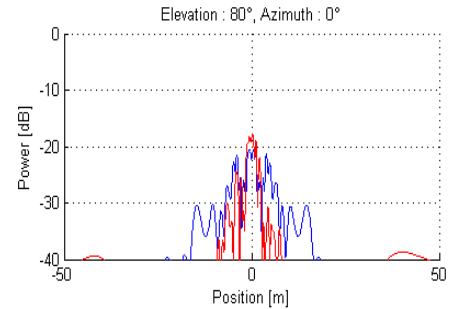

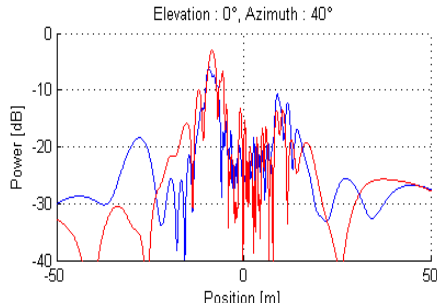

Elevation : $40^{\circ}$, Azimuth : $40^{\circ}$

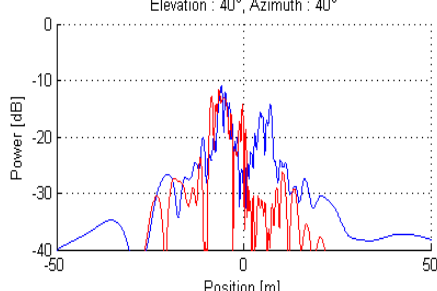

Elevation : $80^{\circ}$, Azimuth : $40^{\circ}$

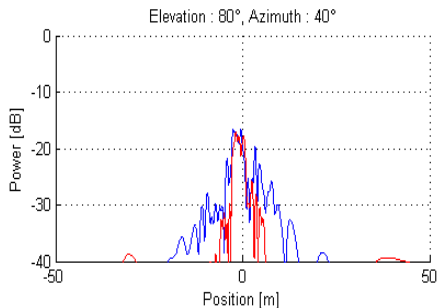

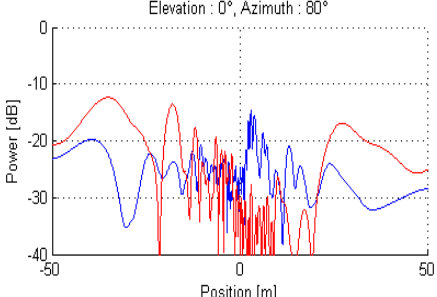

Elevation : $40^{\circ}$, Azimuth : $80^{\circ}$

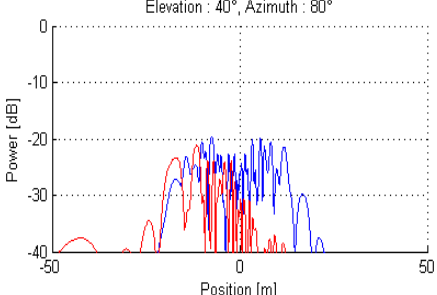

Elevation : $80^{\circ}$, Azimuth : $80^{\circ}$

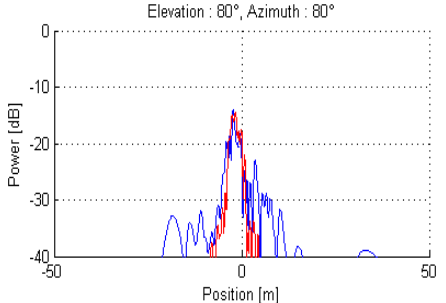

Figure 8. Validation results for city centre building type in various incidence configurations 


\section{SCATTERING OF ISOLATED BUILDING}

This section provides one validation example of the actual implementation of the TCM. The ONERA MoM tool Elsem3D is here used as a reference for this validation example. The simulation context is the following: A mobile receiver is linearly moving at $\mathrm{Z}=0 \mathrm{~m}$ height, following the $\mathrm{Y}$ axis from $\pm 100 \mathrm{~m}$ at a distance of $\mathrm{X}=10 \mathrm{~m}$ from a complex façade. The bottom centre of this façade is placed at the origin $(0,0,0)$. The geometry of the façade represents a city centre building type visible in Figure 3 and detailed in [8].

Diagram in top left of Figure 7 represents the surface currents calculated using Elsem $3 \mathrm{D}$ on a precise mesh of the façade (around $\lambda / 10$ ). The top right diagram of Figure 7 represents the same façade using the TCM. The red parts are specular elements, the blue parts are backscattering elements and the green contours delimit where incoherent scatterers (green points) are randomly spread. Using the TCM, the complex city centre building type is reduced to 20 rectangular zones. The bottom of Figure 7 represents the normalised power scattered by the façade considering a transmitting source situated at $2010^{6} \mathrm{~m}$, elevation $0^{\circ}$, and azimuth $40^{\circ}$. As visible in this plot, the total scattered power calculated using the TCM (plain red curve) presents a very good agreement considering the reference made using Elsem3D (plain blue curve) which is an exact method. The total scattered power is also divided following the three components which are represented using dashed lines. As expected, the contribution of the specular component is predominant in the specular zone around $-10 \mathrm{~m}$ and then rapidly decreases. The backscattering component is predominant for positive $\mathrm{Y}$ with a peak around $+10 \mathrm{~m}$ while the incoherent scattering is predominant for negative $\mathrm{Y}$.

Figure 8 presents a generalised version of Figure 7 for various incidences. The context was kept constant except for transmitter incidence. On Figure 8, the total scattered power is presented considering only the total TCM (red) and the reference Elsem3D (blue). In the following, all nine incidence cases will be denoted using the convention \{elevation,azimuth\}. Note that the distance scale has been reduced to $\pm 50 \mathrm{~m}$ around the façade and the power scale from 0 to $40 \mathrm{~dB}$. This truncation is representative of the interest zone where the TCM will be used in a final application. About the power truncation, considering that a significant MP is visible from a receiver point of view up to $-20 \mathrm{~dB}$ w.r.t. the LOS signal, the TCM model will be analysed on the interval 0 to $-20 \mathrm{~dB}$. If NLOS and blockage conditions occurs $(-20 \mathrm{~dB})$, the model will be analysed up to $-40 \mathrm{~dB}$ w.r.t to the unblocked LOS.

As visible for $\{0,0\},\{0,40\},\{40,0\}$ cases, the actual implementation of the TCM is suitable for LOS and NLOS conditions with very good results w.r.t the reference. For $\{0,80\},\{40,40\},\{40,80\}$ cases, the TCM should be suitable for LOS and NLOS conditions. However, in the actual implementation of the TCM, the backscattering component tends to highly underestimate the second peak in positive $\mathrm{Y}$. For $\{80,0\},\{80,40\},\{80,80\}$ cases, the TCM is suitable for LOS conditions only since only the maximum due to the specular component seems to be well reproduced while the influence zone of the façade is not. Nevertheless, for extreme elevation, the probability of having NLOS conditions and blockages is highly reduced.

\section{CONCLUSION}

In this paper, a new EM simplified model for building scattering has been presented. It is called the Three Component Model (TCM). Observing simulation results and measurement campaigns the total power scattered by a complex façade has been reduced to the sum of three propagation phenomena. The first one is the specular reflection and is originating from wide and smooth surfaces. The second is backscattering and represents a dihedral effect taking place on window corners. The third one is called incoherent scattering and represents the omnidirectional contribution of all small features present on complex façades.

One validation example has been provided using a MoM based tool as a reference. For typical LMS incidences the TCM presents very good results in the range of 0 to $-40 \mathrm{~dB}$ compared to LOS power. However, in the actual implementation of the TCM, the backscattering component presents some failings and must be revised to improve TCM prediction accuracy. This version of the TCM will be soon upgraded to take into account polarisation and dielectric materials. The final application being wideband satellite communications and navigation, MP delay and AoA will also be treated.

\section{ACKNOWLEDGEMENT}

This activity is funded by ONERA Toulouse (Office National d'Etudes et de Recherches Aéronautiques), CNES (Centre National d'Etudes Spatiales) and Thales Alenia-Space.

\section{REFERENCES}

[1] Loo, "A statistical model for a land mobile satellite link," IEEE Transactions on Vehicular Technology, vol. 34, pp. 122-127, 1985.

[2] F.P. Fontan and al., "Statistical modeling of the LMS channel," IEEE Transactions on Vehicular Technology, vol. 50, pp. 1549-1567, 2001.

[3] COST Action 255 - Radiowave Propagation Modelling for SatCom Services at $\mathrm{Ku}$-Band and Above, Final report, March 2002.

[4] C. Oestges and al., "Physical statistical modelling of the land mobile satellite channel based on ray tracing," IEEE Proc. on Microwaves, Antennas and Propagation, vol. 146, pp. 45-49, 1999.

[5] ITU, ITU-R P.681-7 Propagation data required for the design of Earthspace land mobile telecommunication systems. ITU, 2009.

[6] Abele et al., "A new physical-statistical model of the land mobile satellite propagation channel," 4th European Conference on Antennas and Propagation (EuCAP), pp. 1-5, 2010.

[7] F.P. Fontan and al., "An enhanced Markov chain based model for the narrowband LMS channel in built-up areas," Int. J. of Satellite Communications and Networking, vol. 23, pp. 111-128, 2005.

[8] M. Ait-Ighil and al., "Simplifying the Propagation Environment Representation for LMS Channel Modelling," Eurasip Journal (review in progress)

[9] M. Ait-Ighil and al., " Channel Modelling in Complex Urban Environments for Testing Multipath Mitigation Methods Enhanced by Antenna Array", ESA Workshop on Radiowave Propagation, ESA/ESTEC, Noordwijk, The Netherlands, 30 Nov-2 Dec 2011.

[10] G.T. Ruck, D.E. Barrick, W.D. Stuart, C.K. Krichbaum, Radar Cross Section Handbook, Ed Plenom press, New-York, 1970.

[11] A. Lehner et A. Steingass, "Time Series Multipath Modeling of Suburban Environments in Landmobile Satellite Navigation ", 2th European Conference on Antennas and Propagation (EuCAP), 2007. 